\title{
Role of impact parameter in branching reactions: Chemical accelerator studies of the reaction $\mathrm{Xe}^{+}+\mathrm{CH}_{4} \rightarrow \mathrm{XeCH}_{3}{ }^{+}+\mathrm{H}$
}

\author{
G. D. Miller, ${ }^{\text {a) }}$ L. W. Strattan, ${ }^{\text {b) }}$ and P. M. Hierl \\ Department of Chemistry, University of Kansas, Lawrence, Kansas 66045 \\ (Received 26 December 1979; accepted 4 April 1980)

\begin{abstract}
Integral reaction cross sections and product velocity distributions have been measured for the ion-molecule reaction $\mathrm{Xe}^{+}\left(\mathrm{CH}_{4}, \mathrm{H}\right) \mathrm{XeCH}_{3}^{+}$over the relative reactant translational energy range of $0.7-5.5 \mathrm{eV}$ by chemical accelerator techniques. The kinematic results indicate that reaction proceeds in a direct manner by a rebound mechanism over the energy range studied, suggesting that this substitution reaction occurs predominantly in small impact parameter collisions. This finding contrasts with the results obtained for the competing reaction, $\mathrm{Xe}^{+}\left(\mathrm{CH}_{4}, \mathrm{CH}_{3}\right) \mathrm{XeH}^{+}$, where the strong forward scattering of the $\mathrm{XeH}^{+}$product indicates that $\mathrm{H}$-atom abstraction occurs primarily in large impact parameter collisions.
\end{abstract}

\section{INTRODUCTION}

An important problem in chemical kinetics is the identification of the factors which determine which products will be formed when a given pair of reactants can form two or more sets of products, as in

$$
\mathrm{A}+\mathrm{B}-\mathrm{C}+\mathrm{D} \text { or } \mathrm{E}+\mathrm{F} \text {, etc. }
$$

One approach has been to assume that the reactants form an intermediate complex, $(\mathrm{AB})^{*}$, and that competi tion between the various reaction channels is deter mined by the relative rates of unimolecular decomposition of the complex to form the various sets of products. If it is further assumed that the lifetime of $(A B)^{*}$ is sufficiently long to permit equilibration of the excess energy among the various internal degrees of freedom, then the relative rates of decomposition can be calculated from statistical considerations. The general conclusion of the statistical theory is that the reaction will predominantly follow the most exoergic channel. ${ }^{1}$

However, statistical theories of unimolecular decomposition would appear to be inapplicable to those reactions which proceed by a direct mechanism rather than by the formation of a long-lived complex because the duration of the collision (usually $\sim 10^{-13} \mathrm{sec}$ ) in these reactions is insufficient for redistribution of energy within the collision complex. For such reactions it has been postulated ${ }^{2}$ that the reaction path is determined by steric factors (i.e., by the impact parameter and the angle of collision). Until recently, however, evidence in support of this hypothesis has been circumstantial in nature, so that it has been impossible to determine rigorously whether a particular yield pattern was due primarily to steric factors or to other considerations, such as bond energies and inertial effects. ${ }^{3}$

The first direct evidence to support the hypothesis that factors such as the impact parameter determine the reaction path followed in a given collision was obtained from

\footnotetext{
a) Present address: Monsanto Mound Laboratory, P.O. Box 32 , Miamisburg, Ohio 45342 .

${ }^{b}$ Present address: Environmental Protection Agency, Surveillance and Analysis Division, Kansas City, Kansas 66115.
}

a chemical accelerator study of reactive scattering in the system $\mathrm{Kr}^{+}+\mathrm{CH}_{4}{ }^{4}$ In the hope of obtaining further information concerning the role of steric factors in direct reactions, we have studied the competing ionmolecule reactions

$$
\begin{aligned}
\mathrm{Xe}^{+}+\mathrm{CH}_{4}-\mathrm{XeH}^{+}+\mathrm{CH}_{3} \\
-\mathrm{XeCH}_{3}^{+}+\mathrm{H} .
\end{aligned}
$$

Reaction cross sections and product velocity vector distributions have been measured at collision energies over the range $0.4-8 \mathrm{eV}(\mathrm{cm})$. The data for Reaction (2). have already been published, ${ }^{5}$ so this paper will present data for Reaction (3) only. The results are similar to those obtained for the $\mathrm{Kr}^{+}+\mathrm{CH}_{4}$ system ${ }^{4}$ and thus support the hypothesis that, in direct reactions, factors such as the impact parameter determine which products will be formed.

Ion-molecule reactions (including charge transfer) in xenon-methane mixtures have prevlously been studied by high-pressure mass spectrometry, ${ }^{8}$ ion cyclotron resonance, ${ }^{7,8}$ and tandem mass spectrometry. 9,10

\section{EXPERIMENTAL}

\section{A. Apparatus}

The instrument used in this study, a single beam, collision chamber type of chemical accelerator (with which product velocity and angular analysis are possible) has been described in detail elsewhere. ${ }^{5,11}$ It should be pointed out, however, that in the present study the $\mathrm{Xe}^{+}$reactant ions were produced by impact of $33 \mathrm{eV}$ electrons, so that nearly all $(98 \%)$ of the $\mathrm{Xe}^{+}$ions are in the ${ }^{2} P$ state and are presumably distributed statistically in a $2: 1$ ratio between the ${ }^{2} P_{3 / 2}$ ground state and the ${ }^{2} P_{1 / 2}$ excited state, which differ in energy by $1.61 \mathrm{eV} .^{12}$

\section{B. Heats of reaction}

Holtz and Beauchamp ${ }^{7}$ have reported a value of 1.87 $\pm 0.35 \mathrm{eV}$ for the methyl cation affinity of $\mathrm{Xe}$. Assuming values of $4.477 \mathrm{eV}$ for $D_{0}\left(\mathrm{H}-\mathrm{CH}_{3}\right)^{13}$ and $9.840 \mathrm{eV}$ for the ionization potential of $\mathrm{CH}_{3},{ }^{12}$ one finds that Reaction (3) is endothermic by $0.32 \pm 0.35 \mathrm{eV}$ for ${ }^{2} P_{3 / 2}$ ground state 
TABLE I. Integral cross sections for the reaction $\mathrm{Xe}^{+}+\mathrm{CH}_{4} \rightarrow \mathrm{XeCH}_{3}^{+}+\mathrm{H}$.

\begin{tabular}{|c|c|c|c|}
\hline $\begin{array}{l}\text { Initial translational } \\
\text { energy, } E_{T}(\mathrm{eV})\end{array}$ & $\begin{array}{l}\text { Relative collection } \\
\text { efficiency, } K_{c} / K_{A}{ }^{a}\end{array}$ & $\begin{array}{l}\text { Transmission } \\
\text { factor, } K(T)^{\mathrm{b}}\end{array}$ & $\begin{array}{l}\text { Integral reaction } \\
\text { cross sections, } \sigma \\
\left(10^{-16} \mathrm{~cm}^{2}\right)^{\mathrm{c}}\end{array}$ \\
\hline 0.81 & 1. 44 & 0.72 & 0.013 \\
\hline 1.08 & 1.77 & 0.77 & 0.033 \\
\hline 1.56 & 2.43 & 0.54 & 0.077 \\
\hline 2.20 & 1.53 & 0.70 & 0.22 \\
\hline 2.39 & 1.77 & 0.83 & 0.23 \\
\hline 2.96 & 1.74 & 0.75 & 0.24 \\
\hline 4.29 & 2.45 & 0.83 & 0.20 \\
\hline 4.38 & 1.28 & 0.77 & 0.23 \\
\hline 5.18 & 1.72 & 0.83 & 0.085 \\
\hline
\end{tabular}

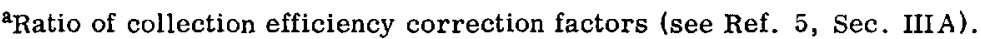

${ }^{b}$ Correction factor for attenuation of primary beam, calculated from Eq. (4) of Ref. 5.

¿Calculated from Eq. (4).

$\mathrm{Xe}^{+}$ions but exothermic by $0.99 \pm 0.35 \mathrm{eV}$ for the ${ }^{2} P_{1 / 2}$ excited state of $\mathrm{Xe}^{+}$.

A similar situation exists for the $\mathrm{H}$-atom abstraction process. Reaction (2) is endothermic by $0.86 \pm 0.11 \mathrm{eV}$ for $\mathrm{Xe}^{+}\left({ }^{2} P_{3 / 2}\right)$ but exothermic by $0.45 \pm 0.11 \mathrm{eV}$ for $\mathrm{Xe}^{+}\left({ }^{2} P_{1 / 2}\right)$.

\section{RESULTS AND DISCUSSION}

\section{A. Reaction cross sections}

Integral reaction cross sections, $\sigma$, for Reaction (3) are calculated from the formula

$$
\sigma=\frac{I_{C} / I_{A}}{n_{B} L} K(T),
$$

where $I_{C}$ is the total reactively scattered product $\left({ }^{132} \mathrm{XeCH}_{3}^{*}\right)$ ion intensity, $I_{A}$ is the primary $\left({ }^{132} \mathrm{Xe}^{*}\right)$ ion intensity, $n_{B}$ is the number density of $\mathrm{CH}_{4}$ in the collision chamber, $L$ is the collision path length, and $K(T)$ is a factor which corrects the thin target formula, $I_{C} / I_{A} n_{B} L$, for any attenuation of the reactant and product beams. ${ }^{14}$ The details of these calculations have been described previously. ${ }^{5,14}$

Table I presents the integral cross sections calculated from $\mathrm{Eq}$. (4), along with the relative collection efficiencies $K_{C} / K_{A}$ and the transmission factor $K(T)$. The uncertainties in the cross sections are estimated to be $\pm 40 \%$ relative to each other and $\pm 60 \%$ on an absolute basis. The excitation function ( $\sigma$ versus collision energy) is presented in Fig. 1.

As shown in Fig. 1, the excitation function for formation of $\mathrm{XeCH}_{3}^{+}$rises from an apparent threshold at a relative collision energy $\left(E_{T}\right)$ of $0.7 \pm 0.2 \mathrm{eV}$ to a maximum in the region $E_{T}=3-4 \mathrm{eV}$, where $\sigma \sim 0.25 \AA^{2}$, and then decreases with increasing collision energy. The lack of data at collision energies above $5 \mathrm{eV}$ is due to the complication in the mass spectrum at higher collision energies because of the formation of $\mathrm{XeCH}_{2}^{+}$and possibly $\mathrm{XeCH}^{+}$ with all possible isotopes of $\mathrm{Xe}$. Below $5 \mathrm{eV}, \mathrm{XeH}^{+}$and $\mathrm{XeCH}_{3}^{+}$are the only detectable products and the data analysis is, therefore, straightforward. The magnitude of the cross section for $\mathrm{XeCH}_{3}^{+}$formation is somewhat larger than that found for $\mathrm{KrCH}_{3}^{+}$formation (about 0.05 $\mathrm{A}^{2}$ at its maximum) in a previous study of the $\mathrm{Kr}^{+}+\mathrm{CH}_{4}$ system, ${ }^{4}$ but is similar in magnitude to that found for $\mathrm{KrCH}_{2}^{+}$formation in that study.

Because the $\mathrm{Xe}^{+}$reactant beam contains ions in both levels of the ${ }^{2} P$ doublet, it is not possible to deduce from the present data whether the two states differ in the rate at which they produce $\mathrm{XeCH}_{3}^{+}$. On the one hand, Reaction $(3)$ is exothermic by $0.99 \pm 0.35 \mathrm{eV}$ for the $\mathrm{Xe}^{+}\left({ }^{2} P_{1 / 2}\right)$ but endothermic by $0.32 \pm 0.35 \mathrm{eV}$ for $\mathrm{Xe}^{+}\left({ }^{2} P_{3 / 2}\right)$. Because most exothermic reactions of positive ions have no activation energy, ${ }^{15}$ the observed threshold energy of $0.7 \pm 0.2 \mathrm{eV}$ could be explained by assuming that the ground state $\mathrm{Xe}^{+}\left({ }^{2} P_{3 / 2}\right)$ ion reacts to form $\mathrm{XeCH}_{3}$ with much greater propensity than does the excited ${ }^{2} P_{1 / 2}$ state, so that the threshold is simply a consequence of the endothermicity for the ground state reactants.

On the other hand, translational energy thresholds have been found for the exothermic reactions $\mathrm{N}_{2}^{+}\left(\mathrm{CH}_{4}\right.$, $\left.\mathrm{CH}_{3}\right) \mathrm{N}_{2} \mathrm{H}^{+16}$ and $\mathrm{Ar}^{+}\left(\mathrm{CH}_{4}, \mathrm{CH}_{3}\right) \mathrm{ArH}^{+14}{ }^{17}$ Although the thresholds found for these reactions $(\approx 0.1 \mathrm{eV})$ are con-

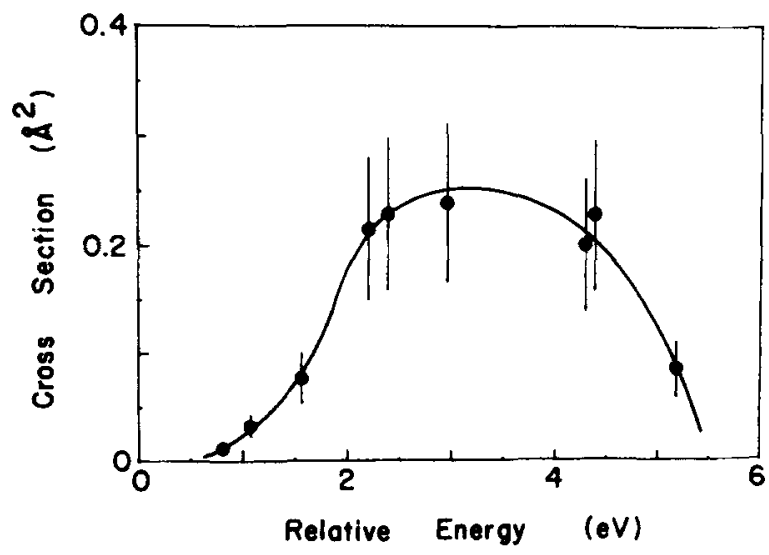

FIG. 1. Integral reaction cross section $\sigma$ vs initial relative translational energy $E_{T}$ for the reaction $\mathrm{Xe}^{+}+\mathrm{CH}_{4} \rightarrow \mathrm{XeCH}_{3}^{+}+\mathrm{H}$ (solid circles). The values of the cross sections, calculated from Eq. (4), are listed in Table I. Estimated experimental uncertainties in $\sigma$ are indicated by error bars. The solid line represents an empirical fit to the experimental data. 


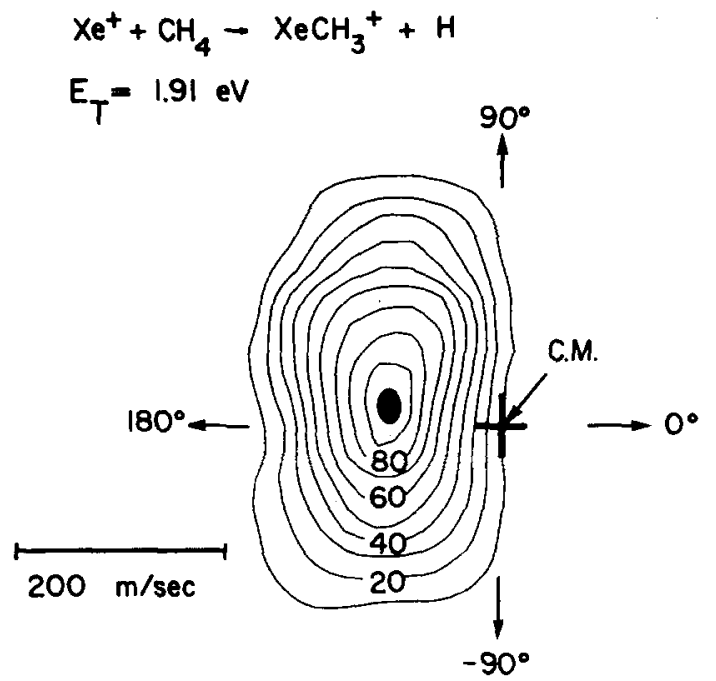

FIG. 2. Product probability distribution for Reaction (3) at the relative collision energy $E_{T}=1.91 \mathrm{eV}$. The product ion intensities, normalized to 100 at the position of maximum intensity, are shown relative to the Cartesian system, $P_{C}$. Arrows represent scattering angle with respect to the center of mass (marked C.M.).

siderably lower than the threshold found for Reaction (3), it is clear that the observation of a translational energy threshold is not sufficient to prove that one is dealing with an endothermic reaction.

\section{B. Kinematics}

As described previously, ${ }^{5}$ the measured product translational energy distributions at various laboratory scattering angles were transformed to probabilities in Cartesian velocity space. ${ }^{18}$ The results are presented as contour maps showing the relative yields of $\mathrm{XeCH}_{3}^{+}$as functions $P_{C}(u, \theta)$ of the c.m. velocity $u$ and scattering angle $\theta$.

Such product velocity vector distributions for $\mathrm{XeCH}_{3}^{+}$ produced at relative collision energies $E_{T}$ of 1.91 and $4.29 \mathrm{eV}$ are shown in Figs. 2 and 3, respectively. At both energies the $\mathrm{XeCH}_{3}^{+}$product is scattered almost entirely in the backward direction (i.e., opposite to the direction of the incident $\mathrm{Xe}^{+}$ion). This asymmetry about the $\theta= \pm 90^{\circ}$ plane is a clear indication that $\mathrm{Re}$ action (3) proceeds in a direct manner (i. $e_{,}$, on a time scale shorter than a rotational period, about $10^{-12} \mathrm{sec}$ ). As reported previously, ${ }^{5}$ the competing $\mathrm{H}$-atom abstraction, Reaction (2), was also found to be dominated by a direct mechanism in this range of collision energies, although a transition to a mechanism involving the formation of a long-lived $\mathrm{XeCH}_{4}^{+}$collision complex was observed in the previous study at relative collision energies less than $0.5 \mathrm{eV}$.

Additional information on the reaction mechanism can be obtained from the product c.m. angular distributions, $I(\theta)$, derived from the product velocity vector distributions (Figs. 2 and 3 ) by the relation

$$
I(\theta)=\int_{0}^{\infty} u^{2} P_{c}(u, \theta) d u
$$

The results, presented as differential polar cross sec- tions, $d \sigma / d \theta=2 \pi I(\theta) \sin \theta$, are shown in Fig. 4. As can be seen, the displacement product $\mathrm{XeCH}_{3}^{+}$is almost entirely scatter ed through angles greater than $90^{\circ}$. This finding contrasts sharply with that for the abstraction process, where the $\mathrm{XeH}^{+}$product is found almost entirely at scattering angles less than $90^{\circ}$ at comparable collision energies. (Compare Fig. 4 with Fig. 9 of Ref. 5.) To the extent that a correlation exists in direct reactions between impact parameter and scattering angle, ${ }^{10}$ we can conclude that the displacement reaction producing $\mathrm{XeCH}_{3}^{*}$ occurs principally in close (i.e., small impact parameter) collisions, while the abstraction reaction producing $\mathrm{XeH}^{+}$occurs predominantly in glancing (i.e., large impact parameter) collisions. Thus, over the energy range considered here, competition between the two reaction channels is apparently governed by the collision geometry.

Similar results have been reported for the analogous reactions of $\mathrm{Kr}^{+}$with $\mathrm{CH}_{4} .^{4}$ As discussed in that paper, the principle of conservation of angular momentum pro$v$ ides a qualitative explanation for this dependence of reaction path upon impact parameter. Reaction will be limited to low angular momentum (i.e., small impact parameter) collisions if (1) the products reduced mass (with respect to translation) is much less than that of the

$$
\begin{aligned}
& \mathrm{Xe}^{+}+\mathrm{CH}_{4} \rightarrow \mathrm{XeCH}_{3}{ }^{+}+\mathrm{H} \\
& E_{T}=4.29 \mathrm{~N}
\end{aligned}
$$

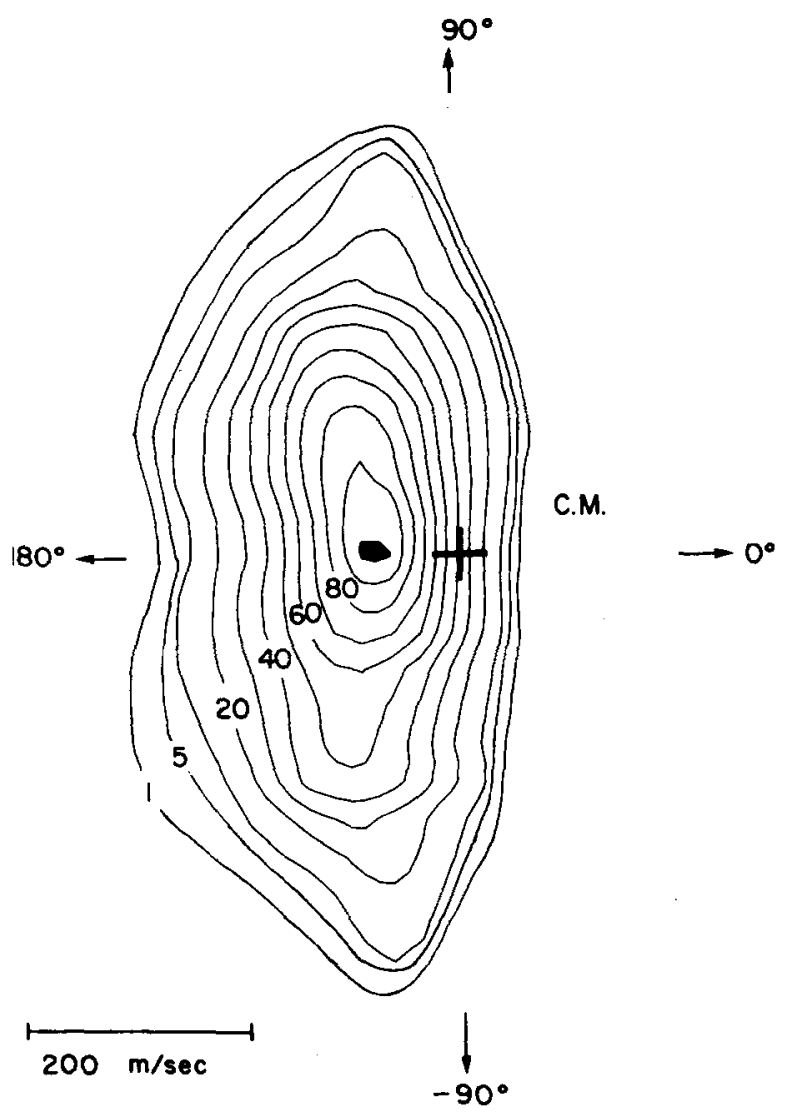

FIG. 3. Product probability distribution for Reaction (3) at $E_{T}=4.29 \mathrm{eV}$. 


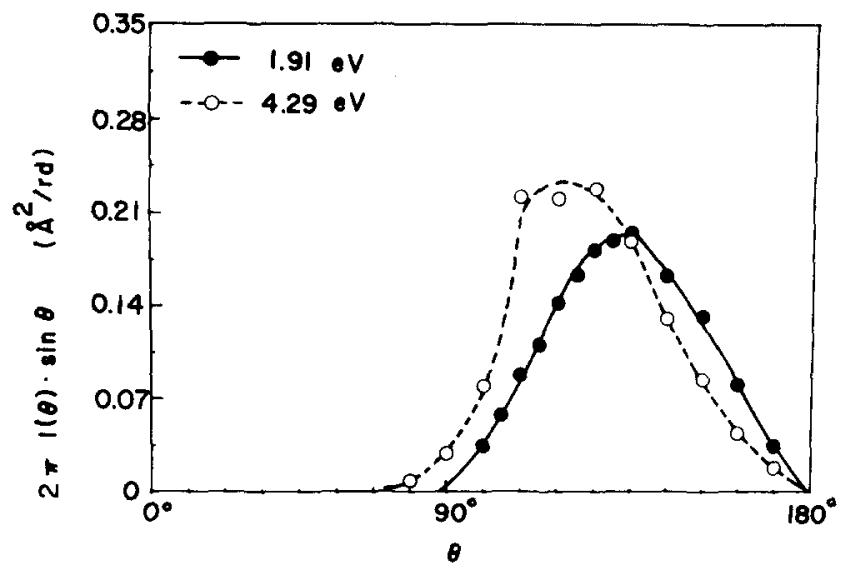

FIG. 4. Polar differential cross sections, $2 \pi I(\theta) \sin \theta$, vs c.m. scattering angle $\theta$ for $\mathrm{XeCH}_{3}^{+}$produced in Reaction (3) at the relative collision energies of 1.91 and $4.29 \mathrm{eV}$. These cross sections were obtained by integrating over the product probability distributions shown in Fig. 2 and 3, using Eq. (5) of the text, and then scaled so that the area under each curve equaled the measured integral reaction cross section at the energy, in accord with the relation $\sigma=2 \pi \int_{0}^{\pi} I(\theta) \sin \theta d \theta$.

reactants, and (2) the intermolecular potential lacks anisotropic terms which could convert reactant orbital to product rotational angular momentum. In the present case $\mu=14.3 \mathrm{amu}$, while $\mu^{\prime}=13.5 \mathrm{amu}$ for the abstraction products, but only $0.99 \mathrm{amu}$ for the displacement products. For abstraction, $\mu^{\prime} \simeq \mu$, and therefore even large amounts of reactant orbital angular momentum can be taken up by the products' orbital rather than rotational motion. Consequently, we can observe $\mathrm{XeH}^{+}$formation from a strippinglike mechanism occurring in glancing collisions. On the other hand, $\mu^{\prime} \ll \mu$ for displacement. $\mathrm{XeCH}_{3}^{+}$formation in large impact parameter collisions would be possible only if large amounts of the reactants' orbital angular momentum could be converted to rotational angular momentum in the products. Evidently this is difficult, because we see $\mathrm{XeCH}_{3}^{+}$produced by a rebound (large scattering angle) mechanism, with an integral cross section much smaller than that for $\mathrm{XeH}^{+}$ production. Both observations indicate that displacement is possible only when the impact parameter (and hence the reactants' orbital angular momentum) is small.

Product velocity distributions, $P(u)$, or relative translational energy distributions, $P\left(E_{T}^{\prime}\right)$, can also be derived from Figs. 2 and 3 by appropriate integration over the scattering angle. Unfortunately, in the present case the mass ratio between the detected $\left(\mathrm{XeCH}_{3}^{+}\right)$product and the undetected $(\mathrm{H})$ product is so large that the recoil velocity $u$ of the detected product is only a very small fraction ( $\left.\frac{1}{\sqrt{48}}\right)$ of the final relative velocity, $u_{R}^{\prime}$. Because of this very unfavorable kinematic factor, even moderately wide spreads in $u_{R}^{\prime}$ arising from a distribution in $E_{T}^{\prime}$ will result in distributions in $u$ that are much narrower than the experimental resolution.

An approximate one-dimensional treatment of the problem demonstrates that nearly all of the observed spread in $\mathrm{XeCH}_{3}^{+}$velocity is caused by the spread in the initial relative velocities arising from the velocity spread of the $\mathrm{Xe}^{+}$reactant beam and the thermal motion of the $\mathrm{CH}_{4}$ target molecules.

As Fig. 5 shows, the distribution $P_{1}\left(v_{1}\right)$ of reactant $\mathrm{Xe}^{*}$ laboratory velocities in the $0^{\circ}$ direction can be fairly well approximated by a Gaussian distribution centered around the most probable $\mathrm{Xe}^{+}$laboratory velocity, $v_{1}^{0}$. The distribution of centroids, $P(V)$, along the $0^{\circ} \mathrm{di}-$ rection, will then also be a Gaussiandistribution centered around $V_{0}$, the nominal velocity of the c.m. [see $E q$. (A5) of the Appendix].

To determine the extent to which this spread in $V$ contributes to the width of the observed $\mathrm{XeCH}_{3}^{+} \mathrm{c} . \mathrm{m}$. velocity distribution, $P(u)$, we have assumed that, at a given collision energy, all $\mathrm{XeCH}_{3}^{+}$product ions actually have the same c.m. velocity, $w_{0}$. As shown in the Appendix, this one-dimensional approximate treatment then predicts that the observed $\mathrm{XeCH}_{3}^{+} \mathrm{c} . \mathrm{m}$. velocity distribution should be of the form

$$
P(u) \propto \exp \left[-\left(\frac{u-w_{0}}{\sigma_{\mathrm{cm}}}\right)^{2}\right] .
$$

Figures 6 and 7 compare the experimental data at $E_{T}$ $=1.91$ and $4.29 \mathrm{eV}$, respectively, with the distributions predicted by $\mathrm{Eq}$. (6), using the parameters given in Table II. As Fig. 6 shows, agreement between the measured distribution and the predicted distribution is excellent at a c,m. scattering angle of $180^{\circ}$, indicating that the shape of the observed distribution is a consequence of the distribution of centroids rather than actual dynamics of the reaction. The only measured quantity carrying any dynamical information, then, is the most probable recoil velocity, which equals (in this approximate treatment) $w_{0}$, the true recoil velocity of $\mathrm{XeCH}_{3}^{+}$. The experimental distributions at smaller angles are skewed

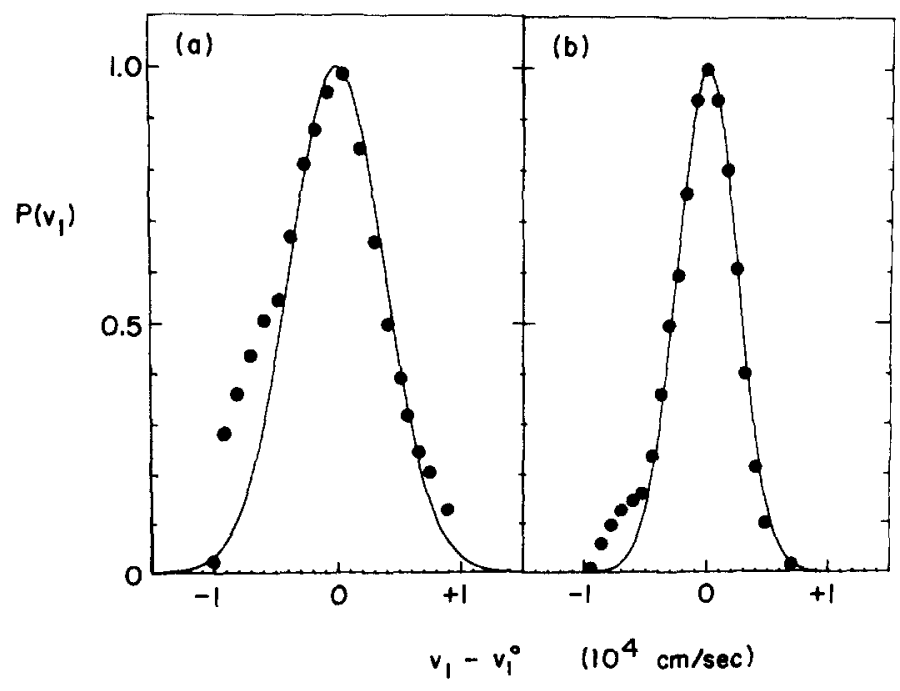

FIG. 5. Distribution $P\left(v_{1}\right)$ of the reactant $\mathrm{Xe}^{+}$ion laboratory velocities along the $0^{\circ}$ axis vs $v_{1}-v_{1}^{0}$, the deviation of the $\mathrm{Xe}^{+}$ laboratory velocity from its nominal value, $v_{1}^{0}$. In panel (a), $E_{T}=1.91 \mathrm{eV}$ and $v_{1}^{0}=5.09 \times 10^{5} \mathrm{~cm} / \mathrm{sec}$; in (b) $E_{T}=4.29 \mathrm{eV}$ and $v_{1}^{0}=7.63 \times 10^{5} \mathrm{~cm} / \mathrm{sec}$. The solid circles represent the measured distribution, and the solid line represents the assumed Gaussian distribution given by Eq. (A4). 


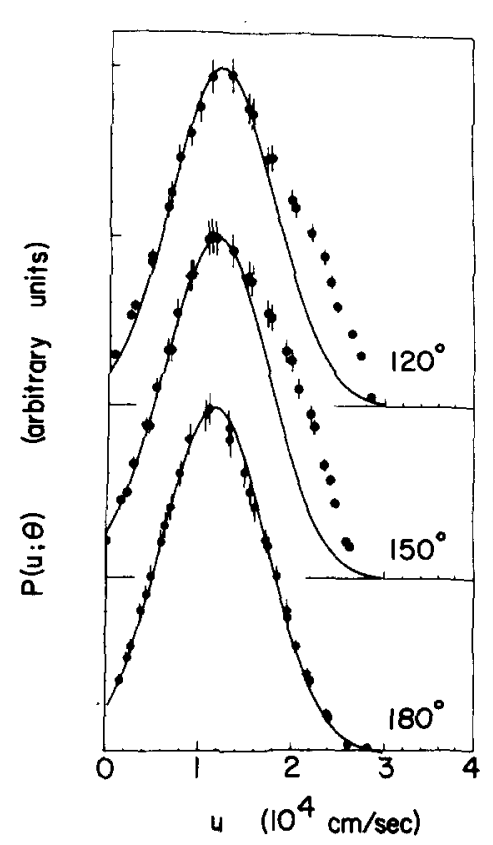

FIG. 6. Distribution $P(u ; \theta)$ of the product $\mathrm{XeCH}_{3}^{+}$ion $\mathrm{c} . \mathrm{m}$. velocities at various c.m. scattering angles for the experiment at a relative collision energy of $1.91 \mathrm{eV}$. The solid circles represent the experimental data, and the solid lines represent the product velocity distributions predicted by Eq. (6).

somewhat to higher velocities than Eq. (6) predicts, but this may well be a consequence of the angular divergence of the $\mathrm{Xe}^{+}$, a factor which was not included in this approximate, one-dimensional treatment.

As shown in Fig. 7, the measured product velocity' distributions at $E_{T}=4.29 \mathrm{eV}$ are somewhat broader than would be expected from just the distribution of centroids,

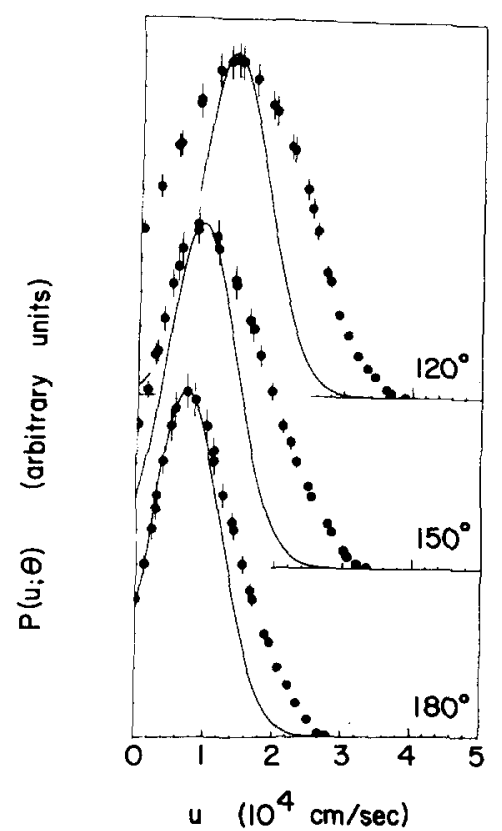

FIG. 7. Distribution $P(u ; \theta)$ of the product $\mathrm{XeCH}_{3}^{+}$ion c.m. velocities at various c.m. scattering angles at $E_{T}=4.29 \mathrm{eV}$. The solid circles represent the experimental data, and the solid lines represent the product velocity distributions predicted by Eq. (6).
TABLE II. Parameters for calculating product velocity distribution, $P(u)$.

\begin{tabular}{lcc}
\hline & $E_{T}=1.91 \mathrm{eV}$ & $E_{T}=4.29 \mathrm{eV}$ \\
\hline$\sigma_{1}\left(10^{4} \mathrm{~cm} / \mathrm{sec}\right)$ & 0.54 & 0.35 \\
$\sigma_{\mathrm{cm}}\left(10^{4} \mathrm{~cm} / \mathrm{sec}\right)$ & 0.81 & 0.72 \\
$\gamma$ & \multicolumn{2}{c}{0.892} \\
$\alpha$ & $6.03 \times 10^{4} \mathrm{~cm} / \mathrm{sec}$ \\
\hline
\end{tabular}

even at $\theta=180^{\circ}$. This indicates that our approximation of the true distribution, $P_{3}(w)$, by a $\delta$ function is no longer valid. Nevertheless, it can be seen that the distribution of centroids is still the dominant factor influencing the shape of the observed distribution, thereby making it very difficult to deduce $P_{3}(w)$ from $P(u)$. Again, the poorer agreement between experiment and Eq. (6) at smaller' scattering angles is presumably a consequence of the approximate treatment employed here.

As the preceding considerations disclose, the peak in the observed product velocity distribution relates directly to the actual reaction dynamics $\left(u_{\mathrm{mp}}=w_{\mathrm{mp}}\right)$, while the shape of $P(u)$ is predominantly a result of the shape of $P(V)$ rather than $P_{3}(w)$. Consequently, as an alternative to the construction of a complete contour map and the subsequent derivation of complete product translational energy distributions, $P\left(E_{T}^{\prime}\right)$, we have simply determined the velocity of maximum product ion intensity in a number of experiments by measuring the product ion translational energy distribution at $0^{\circ}$ in the laboratory and then converting this distribution to the corresponding Cartesian velocity spectrum by multiplying the intensity at each energy by the overall Jacobian factor of $1 / v$. The velocity corresponding to the peak in this velocity spectrum was then used to calculate the most probable value of the translational exoergicity, $Q_{\mathrm{mp}}=E_{T, \mathrm{mp}}^{\prime}$ $-E_{T, \mathrm{mp}}$. The values so obtained, as well as those derived from that data shown in Figs. 2 and 3, are plotted versus $E_{T}$ in Fig. 8. For comparison, values of $Q_{m p}$ taken from Ref. 5 for the abstraction process yielding $\mathrm{XeH}^{*}$ are also shown in $\mathrm{Fig} .8$.

As can be seen, $Q_{m p}$ for $\mathrm{XeCH}_{3}^{+}$is more negative than that for $\mathrm{XeH}^{+}$at the same collision energy. Since, by conservation of energy, the internal energy of the products is given by

$$
E_{I}^{\prime}=E_{I}+\left(-\Delta E_{0}\right)-Q,
$$

we see that displacement products are more excited internally than the abstraction products formed at the same collision energy, and that the internal excitation of the former increases more rapidly with increasing $E_{T}$ than that of the latter. This is just what one would expect if, as suggested earlier, abstraction occurs predominantly in glancing collisions while displacement requires a more nearly head-on collision. The larger fraction of $E_{T}$ directed along the line of centers in the latter situation could be expected to excite more ef fectively the internal modes of the products.

One of several types of collision models that could 




FIG. 8. Most probable translational exoergicity, $Q_{m p}$, vs relative collision energy $E_{T}$ for the reactions $\mathrm{Xe}^{+}+\mathrm{CH}_{4} \rightarrow \mathrm{XeH}^{+}+\mathrm{CH}_{3}$ (open circles) and $\mathrm{Xe}^{+}+\mathrm{CH}_{4}-\mathrm{XeCH}_{3}^{+}+\mathrm{H}$ (solid circles). The dashed line represents the prediction of the ideal knockout model as given by Eq. (9) for the reaction yielding $\mathrm{XeCH}_{3}^{+}$.

produce the back-scattering we see here for $\mathrm{XeCH}_{3}^{+}$is the billard-ball or knockout (IK) process. ${ }^{20-23}$ In this model the $\mathrm{Xe}^{+}$projectile ion would collide impulsively with one hydrogen atom, ejecting it from the $\mathrm{CH}_{4}$ molecule. The $\mathrm{Xe}^{*}$ ion, its velocity diminished, would then pick up the remaining $\mathrm{CH}_{3}$ group to form $\mathrm{XeCH}_{3}^{+}$. Such a process will presumably occur with greatest probability when the axis of the $\mathrm{C}-\mathrm{H}$ bond is perpendicular to the velocity vector of the projectile ion and the impact parameter is approximately equal to one-half the $\mathrm{C}-\mathrm{H}$ bond distance.

For the general reaction $\mathrm{X}^{+}+\mathrm{YZ} \rightarrow \mathrm{XY}^{+}+\mathrm{Z}$, the kinematics of the IK model predicts that, for $180^{\circ}$ scattering, the translational exoergicity should be the negative of the translational energy of the reactant ion $X^{+}$relative to the abstracted group $\mathrm{Y}$ after the ion has collided elastically with the ejected species $Z$ :

$$
Q_{\mathrm{IK}}=-\frac{(Y)(X-Z)^{2}(X+Y Z)}{(X+Y)(X+Z)^{2}(Y Z)} E_{T},
$$

where $(Y)$ represents the mass of $Y$, etc. For Reaction (3), with the masses of $\mathrm{X}, \mathrm{Y}$, and $\mathrm{Z}$ equal to 132,15 , and $1 \mathrm{amu}$, respectively, the IK model predicts $Q_{\mathrm{IK}}$ $=-0.916 E_{T}$. This prediction is shown by the dashed line in Fig. 8.

We find that the IK model overestimates product internal excitation (i.e., $Q_{m p}$ is less negative than $Q_{I K}$ ) at relative collision energies less than $4 \mathrm{eV}$. Previous applications of the IK model to the $\mathrm{T}-\mathrm{CH}_{4},{ }^{2 \mathrm{~b}} \mathrm{O}^{+}-\mathrm{H}_{2},{ }^{23}$ and $\mathrm{N}_{2}^{+}-\mathrm{H}_{2}$ (Ref. 24) systems have also been unsatisfactory, and reasons for such failure have been discussed. ${ }^{23}$ At collision energies above $4 \mathrm{eV}$, however, the IK model much more nearly predicts the observed $Q_{m p}$. The improved agreement at high energy would seem to be in accord with one's chemical intuition. At high collision energies, the reaction dynamics should be dominated more by the steep repulsive part of the system's poten- tial energy surface and be less affected by the attractive part.

\section{SUMMARY AND CONCLUSIONS}

(1) In the $\mathrm{Xe}^{+}-\mathrm{CH}_{4}$ system, the displacement reaction producing $\mathrm{XeCH}_{3}^{*}$ is only a minor reaction channel. The reaction cross section has an apparent translational energy threshold of $0.7 \pm 0.2 \mathrm{eV}$, passes through a maximum of about $0.25 \AA^{2}$ in the region $E_{T}=3-4 \mathrm{eV}$, and then decreases with increasing collision energy.

(2) The $\mathrm{XeCH}_{3}^{+}$velocity vector distributions at $E_{T}$ $=1.91$ and $4.29 \mathrm{eV}$ are asymmetric about the c. m., indicating that reaction proceeds by a direct mechanism over this energy range, as was found $d^{5}$ for the abstraction product $\mathrm{XeH}^{+}$at relative collision energies above 0.5 $\mathrm{eV}$.

(3) The $\mathrm{XeCH}_{3}^{+}$product is scattered through large angles with high internal excitation. In contrast, the $\mathrm{XeH}^{*}$ product is scattered through small angles with relatively low product excitation. This implies that the opacity functions (reaction probability as a function of impact parameter) are significantly different for the two reactions, with abstraction being favored by moderately large impact parameters and displacement being most likely in small impact parameter collisions. This interpretation is consistent with the need to conserve angular momentum and the difficulty of doing so when the reduced mass of the products is much less than that of the reactants.

\section{ACKNOWLEDGMENTS}

Acknowledgment is made to the University of Kansas General Research Fund for support of this research. We wish to thank Professor R. Sampson and the Kansas Geological Survey for providing the computer program used to construct the intensity contour diagrams.

\section{APPENDIX}

If $v_{1}$ and $v_{2}$ are the laboratory velocities of the ionic and neutral reactants, respectively, the velocity $V$ of the center of mass is given by

$$
V=\gamma v_{1}+(1-\gamma) v_{2}
$$

where $\gamma=m_{1} /\left(m_{1}+m_{2}\right), m_{1}$ and $m_{2}$ being the masses of the ionic and neutral reactants, respectively.

If $P_{1}\left(v_{1}\right)$ and $P_{2}\left(v_{2}\right)$ represent the distributions in ionic and neutral reactant velocities, the distribution in $V$ is found by convoluting $P_{1}\left(v_{1}\right)$ with $P_{2}\left(v_{2}\right)$, subject to the condition that $v_{2}=\left(V-\gamma v_{1}\right) /(1-\gamma)$ :

$$
P(V)=\int_{-\infty}^{\infty} P_{1}\left(v_{1}\right) \cdot P_{2}\left(\frac{V-\gamma v_{1}}{1-\gamma}\right) d v_{1} .
$$

In the approximate one-dimensional treatment employed here, the distribution of target velocities along the $0^{\circ}$ direction is given by the one-dimensional Maxwellian distribution function,

$$
P_{2}\left(v_{2}\right) \propto \exp \left[-\left(\frac{v_{2}}{\alpha}\right)^{2}\right],
$$


where $\alpha=\left(2 k T / m_{2}\right)^{1 / 2}, k$ being the Boltzmann constant and $T$ the target gas temperature. If the distribution of reactant ion velocities in the $0^{\circ}$ direction is assumed to be a Gaussian distribution centered around the most probable ionic velocity, $v_{1}^{0}$, we have

$$
P_{1}\left(v_{1}\right) \propto \exp \left[-\left(\frac{v_{1}-v_{1}^{0}}{\sigma_{1}}\right)^{2}\right],
$$

where $\sigma_{1}$ is related to the measured full width of the ionic velocity distribution at half its maximum (FWHM) by $\sigma_{1}=$ FWHM $/ 2 \sqrt{\ln 2}$.

Substitution of Eqs. (A3) and (A4) into (A2), followed by integration of the resulting formula, produces the result

$$
P(V) \propto \exp \left[-\left(\frac{V-V_{0}}{\sigma_{\mathrm{cm}}}\right)^{2}\right],
$$

where $V_{0}$, the nominal velocity of the c.m., is given by $\gamma v_{1}^{0}$, and

$$
\sigma_{\mathrm{cm}}^{2}=\gamma^{2} \sigma_{1}^{2}+(1-\gamma)^{2} \alpha^{2} .
$$

If a reactant pair whose $c . m$. has velocity $V$ produces an ionic product whose true recoil velocity in the c.m. system is $w$, this product will be detected at a laboratory velocity $v_{3}=V+w$. If, in interpreting the data, we assume that the velocity of the c.m. is simply $V_{0}$ for all reactant pairs, we would attribute a c.m. recoil velocity $u=v_{3}-V_{0}$ to the ionic product detected at laboratory velocity $v_{3}$. Thus, the assumed recoil velocity $u$ is related to the true recoil velocity $w$ by

$$
u=w+\left(V-V_{0}\right) \text {. }
$$

Since any combination of $w$ and $V$ satisfying Eq. (A7) for a given value of $u$ would contribute to the measured product intensity at $u$, the observed product c.m. velocity distribution $P(u)$ is the result of convoluting the true (but unknown) product velocity distribution $P_{3}(w)$ with the distribution of centroids, $P(V)$, subject to the condition expressed in Eq. (A7);

$$
P(u)=\int_{-\infty}^{\infty} P_{3}(w) \cdot P\left(V=V_{0}+u-w\right) d w .
$$

It is hoped that $P(V)$ is much narrower than $P_{3}(w)$. In the limit that $P(V)$ is a $\delta$ function located at $V=V_{0}$, we have the happy result that the observed and the true distributions are identical, $\boldsymbol{P}(u)=P_{3}(w)$.

Unfortunately, the unfavorable kinematic factor in the present situation probably causes $P_{3}(w)$ to be much narrower than $P(V)$. The limit in this case would be a situation where all the ionic products have the same c.m. recoil velocity $w_{0}$. Then

$$
\begin{aligned}
P_{3}(w)=\delta\left(w_{0}\right)=1 & \text { if } w=w_{0} \\
0 & \text { if } w \neq w_{0} .
\end{aligned}
$$

Substitution of this assumed product velocity distribution into $\mathrm{Eq}$. (A8) yields

$$
\begin{aligned}
P(u) & =P\left(V=V_{0}+u-w_{0}\right) \\
& \propto \exp \left[-\left(\frac{u-w_{0}}{\sigma_{\mathrm{cm}}}\right)^{2}\right]
\end{aligned}
$$

That is, the observed c.m. velocity distribution for the ionic product would be a Gaussian distribution centered around the true c.m. recoil velocity $w_{0}$ and having a width equal to that of the distribution of centroids.

'See, for example, R. D. Levine and R. B. Bernstein, Molecular Reaction Dynamics (Oxford University, New York, 1974), pp. 221-227.

2(a) R. Wolfgang, Annu. Rev. Phys. Chem. 16, 15 (1965); (b) Prog. React. Kinet. 3, 97 (1965).

${ }^{3}$ (a) J. W. Root, W. Breckenridge, and F. S. Rowland, J. Chem. Phys. 43, 3694 (1965); (b) E. Tachikawa, Y. -N. Tang, and F. S. Rowland, J. Am. Chem. Soc. 90, 3584 (1968); (c) E. Tachikawa and F. S. Rowland, ibid. 90, 4767 (1968); (d) ibid. 91, 559 (1969); (e) T. Tominaga and F. S. Rowland, J. Phys. Chem. 72, 1399 (1968).

${ }^{4} J$. R. Wyatt, L. W. Strattan, and P. M. Hierl, J. Chem. Phys. 63, 5044 (1975); J. Phys. Chem. 80, 2911 (1976).

${ }^{5}$ G. D. Miller, L. W. Strattan, C. L. Cole, and P. M. Hierl, J. Chem. Phys. 74, 5082 (1981).

${ }^{6}$ J. L. Franklin and F. H. Field, J. A m. Chem. Soc. 83, 4509 (1961).

${ }^{7}$ D. Holtz and J. L. Beauchamp, Science 173, 1237 (1971).

${ }^{8} \mathrm{~J}$. H. Futrell and R. P. Clow, J. Chem. Phys. 50, 5041 (1969).

${ }^{9}$ T. O. Tiernan and P. S. Gill, J. Chem. Phys. 50, 5042 (169). (1969).

${ }^{10}$ A. J. Masson, Ph.D. thesis, Brandeis University, 1970.

${ }^{11}$ P. M. Hierl, L. W. Strattan, and J. R. Wyatt, Int. J. Mass Spectrom. Ion. Phys. 10, 385 (1973).

${ }^{12}$ Natl. Bur. Stand. Ref. Data Ser. 26 (1969).

${ }^{13}$ W. A. Chupka, J. Chem. Phys. 48, 2337 (1968).

${ }^{14} \mathrm{~J}$. R. Wyatt, L. W. Strattan, S. Chivalak, and P. M. Hierl, J. Chem. Phys. 63, 4582 (1975).

${ }^{15}$ F. W. Lampe, J. L. Franklin, and F. H. Field, Prog. React. Kinet. 1, 84 (1961).

${ }^{16}$ J. R. Wyatt, L. W. Strattan, S. C. Snyder, and P. M. Hierl, J. Chem. Phys. 64, 3757 (1976).

${ }^{17} \mathrm{~J}$. R. Wyatt, L. W. Strattan, S. C. Snuder, and P. M. Hierl, J. Chem. Phys. 60, 3702 (1974); 62, 2555 (1975).

${ }^{18} \mathrm{R}$. Wolfgang and R. T. Cross, J. Phys. Chem. 73, 743 (1969); P. M. Hierl, Z. Herman, and R. Wolfgang, J. Chem. Phys. 53, 660 (1970).

${ }^{19} \mathrm{See}$, for example, Ref. 1, pp. $76-77$

${ }^{20}$ W. F. Libby, J. Am. Chem. Soc. 69, 2523 (1947).

${ }^{21}$ R. J. Cross and R. Wolfgang, J. Chem. Phys. 35, 2002 (1961).

${ }^{22}$ J. C. Light and J. Horrocks, Proc. Phys. Soc. London 84, 527 (1964).

${ }^{23}$ K. T. Gillen, B. H. Mahan, and J. S. Winn, J. Chem. Phys. 58,5373 (1973).

${ }^{24}$ W. R. Gentry, E. A. Gislason, B. H. Mahan, and C. W. Tsao, J. Chem. Phys. 48, 3058 (1968). 